\title{
Les locals et la mondialisation ou des difficultés pour appréhender une réalité spatiale protéiforme
}

\section{Globalization and diversity of local territories: strategies and adaptations}

\author{
Franck Chignier-Riboulon*, Mauricette Fournier** \\ maîtres de conférences en géographie à l'université Blaise Pascal \\ de Clermont-Ferrand
}

\author{
CERAMAC \\ 29 boulevard Gergovia, \\ 63037 Clermont-Ferrand cedex 1 - France
}

\begin{abstract}
Résumé
Derrière l'apparente uniformisation qui semble prévaloir derrière la globalisation, la diversité des situations locales indique que les formes d'adaptation sont variées. En effet, pour affronter la concurrence mondiale, chaque ensemble économique développe des stratégies spécifiques, entre partenariats et coopérations diverses. Les cinq cas présentés (situés en France, Italie et Portugal) illustrent les actions des acteurs locaux pour développer l'économie locale et préserver les emplois. Pour autant, la situation passée a pu être difficile et les investissements récents n'empêchent pas une certaine fragilité.
\end{abstract}

(C) 2006 Lavoisier, Paris. Tous droits réservés.

Adresses email : *Franck.CHIGNIER-RIBOULON@univ-bpclermont.fr,

**Mauricette.FOURNIER@univ-bpclermont.fr

(c) 2006 Lavoisier, Paris. Tous droits réservés. 


\section{Summary}

The process of globalisation has frequently prompted local strategies to develop a 'cluster' approach in response to increased international economic competition. However, each cluster is characterised by specific policies, partnerships and patterns of co-operation to promote competitiveness. This paper presents five case studies (located in France, Italy and Portugal) that illustrate the actions of local policy makers and other stakeholders in seeking to promote and safeguard economic activity and employment. Each locality has experienced economic decline in the recent past and their local economies exhibit continuing weakness, notwithstanding recent innovative policies.

(C) 2006 Lavoisier, Paris. Tous droits réservés.

Mots clés : districts industriels, développement local, stratégies partenariales et adaptation

Keywords: clusters, local development, local partnership.

La mondialisation et ses effets (polarisation, concentration des fonctions de commandement...) sont bien une réalité et une réalité qui s'accélère. Néanmoins, le local perdure, revêtant des figures multiples, parfois animées (ou réanimées) par l'action publique. Autrement dit, la coexistence de deux logiques de production ne signifie pas pour autant, bien au contraire, une imperméabilité de l'une à l'autre. La mondialisation exprime une nouvelle forme des concurrences sectorielles et spatiales. Dans ce cadre, le local ou plutôt les locals se positionnent plus ou moins bien dans la hiérarchie mondiale de production de valeurs ajoutées. Dans l'imagerie commune, ce local renvoie bien souvent à des représentations d'ensembles urbains de petite taille ou à des espaces ruraux plus ou moins post-agricoles, mais il peut tout aussi bien s'agir de métropoles affirmées, ou d'espaces infra-métropolitains.

Or, comme tout élément vivant, l'espace économique, même territorialisé, naît, grandit, s'adapte (ou non), se transforme et connaît une durée de vie plus ou moins longue. La diversité des situations, dans le temps et l'espace, nous amène à proposer le vocable « les locals ». Dans ces conditions, existe-t-il véritablement une logique modélisable qui tendrait à rapprocher telle situation d'un phénomène de mondialisation, ou bien d'une dynamique locale opérante ou, enfin, puisque c'est la mode, d'une glocalisation nouvelle venue ? Certes, chaque situation peut être inscrite dans un type d'évolution donné. Néanmoins, le local connaît une fragilité permanente (et souhaitable ?) contrebalancée par bien des ressorts (co-opétition, fierté locale, logiques de préservation, acteurs providentiels, capacité à révéler des innovations sociales et économiques,...). De fait, l'enjeu principal est la pérennisation de l'économie locale, sous une forme ou une autre. Dans cette perspective, la capacité des réseaux apparaît essentielle. Ces réseaux, locaux avant tout, mais pas uniquement, permettent de révéler les besoins économiques à venir et les potentialités du local à les saisir. En définitive, la capacité du local n'est pas réductible à une forme, elle se traduit par des formes d'auto-organisation, de lobbying, d'adaptation des politiques publiques... qui donnent toute leur autonomie aux cas présentés ci-après. 


\section{Lumezzane et l'agence Lumetel : dynamiques et capacités d'auto-organisation d'un district au-delà des reconnaissances officielles formelles}

\subsection{La reconnaissance des districts : les avancées législatives}

Bien que les districts italiens soient considérés depuis une vingtaine d'années comme exemplaires des nouvelles dynamiques à l'œuvre (Benko, 1992 et 2000), le modèle économique fondé sur les grappes de petites entreprises a été long à s'affirmer, y compris en Italie. Durant la période du « miracle économique » (1946-1973), l’État italien a favorisé le développement des grandes entreprises, privées et surtout publiques, auxquelles il a accordé un important soutien financier (Vidal, 1998). Les districts industriels ne suscitaient alors guère d'intérêt. La crise qui a particulièrement affecté les grandes régions industrielles et les grandes firmes fonctionnant sur le modèle fordiste a conduit, dans le courant des années 80 , à reconsidérer (tout d'abord en Italie, puis un peu partout dans le monde), le rôle des PMI, jusqu'alors bien négligées, et à redécouvrir deux facteurs oubliés par les théoriciens de l'économie classique : le territoire et l'entrepreneur, cher à Schumpeter'. S'appuyant sur l'ancien concept marshallien de « district », Giacomo Becattini (1989) a redécouvert l'intérêt de ces agglomérations d'entreprises, définissant le district comme « une entité socio-territoriale caractérisée par la présence d'une communauté de personnes et d'une population d'entreprises industrielles dans un espace géographique et historique donné ».

Cette nouvelle vision de la politique industrielle, maintenant assez largement partagée, s'inscrit dans une réflexion mondiale sur les nouvelles formes de l'économie (Datar-OCDE, 2001). Partout, ces grappes d'entreprises (districts ou SPL ${ }^{2}$ ) suscitent l'intérêt des pouvoirs publics, qui cherchent à mettre en place des politiques d'appui propres à renforcer la compétitivité des territoires dans le grand jeu de la mondialisation. Ainsi l'OCDE a-t-elle par exemple organisé à Bologne, en juin 2000, une conférence ministérielle qui a débouché sur l'adoption d'une «Charte OCDE sur les politiques à l'égard des $P M E »$. Cependant, si les institutions nationales ont en général pris conscience que l'organisation productive sous forme de grappes d'entreprises n'était pas un phénomène économique marginal, mais représentait une véritable voie de développement, les formes de reconnaissance de ces territoires économiques restent, bien qu'à des degrés divers, encore assez timides.

Or, si les districts italiens représentent une part considérable de l'économie nationale $e^{3}$ ils n'ont été que tardivement pris en considération par l'État. Le système juridique italien ne reconnaissait pas d'existence administrative aux agglomérations de PME-PMI avant la loi 317 de 1991 sur le développement et l'innovation des petites entreprises. L'article 36 de cette loi tente de proposer une définition juridique des districts industriels, décrits comme des "aires territoriales localisées, caractérisées par une concentration élevée de petites entreprises spécialisées, entretenant un rapport ténu avec la population

1 Joseph Schumpeter, 1942, Capitalisme, socialisme et démocratie, Payot.

2 SPL, Système productif local.

3 En 2001, l'Institut des statistiques (ISTAT) a identifié 199 districts, regroupant environ 239000 entreprises manufacturières (40\% du total italien), qui employaient 2,2 millions de personnes (45\% de l'emploi de ce secteur). 
locale »(Vidal, 2001). Cette politique s'inscrivait dans le cadre de la décentralisation des pouvoirs de l'État vers les Régions (transfert des compétences économiques). La loi 317/91 prévoyait ainsi que les Régions pouvaient participer au financement des projets innovants, sur la base d'un contrat de programme entre elles et les consortia (entités économiques publiques). Cependant cette initiative législative n'a pas connu un véritable succès : en 1998, peu de Régions (9 sur 22) avaient exercé leurs compétences dans ce cadre. En 1999, la loi 140, venue préciser les relations entre Régions et districts, ainsi que la définition de ces derniers, n'a à son tour pas été suivie de beaucoup d'effets. Une étude, réalisée en 2002 par l'Institut pour la Promotion Industrielle (IPI), a montré que les engagements réalisés par les Régions en faveur des districts s'étaient en fait limités à la délimitation territoriale et à la création de réglementations pour gérer les interventions. Ainsi, malgré le dispositif législatif dont s'est dotée l'Italie afin de conforter la compétitivité et l'innovation de ses PMI regroupées dans des districts, les politiques publiques peinent à trouver les instruments adaptés à la réalité, si bien que l'on observe un permanent déséquilibre entre les mesures formelles (programmes, études) et les véritables interventions en direction des entreprises. En contre-point de ces hésitations en matière de politique publique, on peut mettre en exergue les dynamiques entrepreneuriales locales et les capacités d'auto-organisation dont ont fait preuve les districts, ainsi qu'en témoigne l'exemple de Lumezzane et son agence de développement Lumetel.

\subsection{Le système productif de Lumezzane}

Situé dans une vallée alpine à une vingtaine de kilomètres au nord de Brescia, Lumezzane constitue un pôle industriel spécialisé dans la production de robinetterie, de vannes et d'articles ménagers qui présente toutes les caractéristiques d'un district industriel marshallien: délimitation territoriale précise, existence d'une communauté fondée sur le partage des mêmes valeurs, tissu économique dominé par les PMI, forte division du travail, étroitesse des relations entre les entreprises, taux de création d'entreprise très élevé. Lumezzane est un district « historique » qui se présentait déjà, à la fin du XVII siècle, comme une aire industrielle bien structurée, spécialisée dans le travail des métaux. La région s'est beaucoup développée au cours des deux guerres mondiales du fait de son orientation vers les industries d'armement et les principaux secteurs de production actuels (robinets et vannes) n'ont émergé que dans l'après-guerre. Le district a alors connu une croissance sans précédent (le nombre d'emplois a doublé dans l'industrie) liée à l'explosion de la demande dans le secteur du bâtiment. Cet espace fortement industrialisé se signale aussi par un cycle de production intégré verticalement, qui va de la fusion du métal jusqu'aux produits finis: sur les 1982 entreprises actives du district, 1059 travaillent dans l'industrie ${ }^{4}$ et occupent 6494 personnes soit environ $80 \%$ de l'ensemble des actifs ; la structure du district montre encore la prédominance des entreprises de petite, voire de très petite taille, la dimension moyenne étant de 10 actifs par établissement et le modèle dominant reste l'entreprise familiale : ainsi, $88 \%$

\footnotetext{
${ }^{4}$ Source : Chambre de Commerce de Brescia, janvier 2001.
} 
des entreprises sont-elles des sociétés de personnes et seulement $12 \%$ des sociétés de capitaux (et parmi celles-ci, le contrôle familial reste majoritaire). Toutefois, si ses résultats économiques apparaissent enviables (le chiffre d'affaires global se situe entre 700 et 900 millions d'euros, pour moitié réalisé grâce aux exportations ${ }^{5}$ ), le district connaît, depuis une douzaine d'années, certaines difficultés qui se sont traduites par la stagnation du nombre d'emploi et un mouvement de concentration des entreprises (les petites unités voient leur nombre se réduire).

\subsection{La consolidation actuelle du district : le rôle de l'Agence LUMETEL}

La globalisation de l'économie s'est en effet traduite pour le district de Lumezzane par l'apparition de nouveaux concurrents, surtout asiatiques, qui ont réussi à conquérir des marchés autrefois dominés par les produits italiens grâce à l'utilisation d'une politique de prix agressive. La compétition est devenue d'autant plus vive que la technologie des produits du district, arrivés à maturité, est devenue accessible à ses concurrents (Chinois notamment). Certains entrepreneurs se sont alors rendus compte qu'ils ne disposaient pas d'armes suffisantes pour affronter de manière adéquate les défis posés par la mondialisation, car ils n'avaient pas accès à l'information concernant les technologies, les marchés, les concurrents, les nouveaux fournisseurs ou les partenaires potentiels. Pour les entrepreneurs, le besoin de nouveaux services s'est rapidement fait sentir, besoin auquel a tout aussi rapidement répondu la commune de Lumezzane. Dès 1986, elle présente, en collaboration avec la Banca Popolare di Brescia, le projet de création d'un centre de services télématiques pour les entreprises. Il s'agit alors d'une véritable innovation car aucune structure de ce genre n'avait encore été créée en Italie.

Face au nouveau contexte économique, plusieurs chefs d'entreprises de Lumezzane se sont aussi associés à la commune afin de créer, en collaboration avec la Région Lombardie, un instrument efficace pour améliorer leur compétitivité tant au plan national qu'international : l'Agence Lumetel. Le premier objectif de l'agence est de permettre aux entreprises d'accéder aux informations nécessaires pour rester compétitives (nouvelles innovations technologiques, données sur les marchés, prix, normes internationales). Ainsi, en disposant de renseignements précis sur l'état actuel de la concurrence et sur les nouvelles opportunités dont elles peuvent disposer, les entreprises peuvent réagir de manière adéquate aux évolutions de l'environnement international. L'information est diffusée notamment par le biais d'un périodique mensuel, Lumetel Notizie, qui propose aux industriels un ensemble de renseignements techniques. Au fil des années, les domaines d'intervention de l'agence ont évolué en fonction de la demande des entreprises. Aujourd'hui Lumetel propose toute une série de services aux PMI : recherche de nouveaux clients, marchés et fournisseurs, analyse de la concurrence, formation professionnelle, contrôle de la qualité, économies d'énergie obtention des labels de qualité, sécurité du travail,...

L'agence Lumetel représente donc une application exemplaire, au niveau local, de la politique industrielle de soutien des entreprises. Cette initiative est née de la volonté de mettre en place un outil adapté à la réalité du district, permettant aux petits entrepreneurs de se mesurer aux «grands » sur le marché mondial. En une décennie, Lumetel, qui

5 Source : Club des Districts Industriels Italiens, 2001 
travaille en étroite collaboration avec les entrepreneurs et les associations locales, s'est véritablement intégrée au territoire. Elle constitue un modèle pour beaucoup de districts, italiens ou étrangers. C'est l'une des raisons pour lesquelles elle est devenue, en 2000, le siège du Club des districts industriels italiens (Club Dei Distretti Industriali). Regroupés au sein de cette association, créée en 1994, les districts italiens oeuvrent pour que l'État les reconnaisse véritablement comme des entités à caractère économique, dotées de personnalité morale, et les considère comme des interlocuteurs privilégiés en matière de politique industrielle et de développement économique, en leur accordant notamment le droit de gérer eux-mêmes les aides. L'un des premiers objectifs du Club, qui rassemblait 27 districts en 2002, consiste donc à soutenir la décentralisation en cours, de l'État vers les Régions et les districts, et tout particulièrement le transfert de compétences en matière de politique industrielle afin que les mesures adoptées soient définies en collaboration avec les acteurs locaux, et réellement adaptées aux besoins de chaque district. Fort de sa représentativité économique, le Club se veut donc l'interlocuteur privilégié des districts face à l'État et aux Régions, avec lesquels il a du reste déjà réalisé un certain nombre d'actions de promotion, en Italie et à l'étranger : collaboration avec l'Institut National du Commerce Extérieur, élaboration d'accords de programmes avec les Régions (prévus par la loi 241 de 1990) pour soutenir l'internationalisation des districts (prospection de marchés pour l'exportation, participation à des foires internationales...).

\section{Thiers : de la fonction avant-gardiste des acteurs publics locaux à la « visibilisation » sous forme de SPL}

\subsection{Transformations et adaptations du système productif}

Situé dans l'est de l'Auvergne, le bassin coutelier thiernois constitue un prototype de district industriel ancré dans une tradition pluriséculaire. Papeteries, tanneries et coutelleries y ont vu le jour avant la fin du Moyen-Âge, favorisées par le courant de relations économiques et politiques avec le sillon rhodanien. La coutellerie, qui ne s'est vraiment imposée qu'au cours du XIX ${ }^{\mathrm{e}}$ siècle quand les activités papetières et textiles ont amorcé leur déclin, a façonné l'identité culturelle de la région. Malgré la crise structurelle qui affecte depuis plusieurs décennies son principal secteur d'activité, la coutellerie, le Pays de Thiers, reste exceptionnellement industrialisé : environ un actif sur deux travaille dans le secteur secondaire. Le bassin a su s'adapter à la mondialisation de l'économie, grâce notamment à la diversification croissante de ses activités qui a pris des formes très variées. La diversification coutelière s'est tout d'abord faite dans les variétés de couteaux et outils coupants, tandis que la papeterie et la gainerie trouvaient une conversion prometteuse dans l'emballage (en carton, en paraffine, en plastique), le conditionnement et les articles de classement. Après 1950, l'apparition de l'acier inoxydable va conduire certains couteliers thiernois à s'orienter vers la platerie. Le remplacement progressif, à la Belle Époque, des matériaux naturels (bois, corne) utilisés pour la fabrication des manches de couteaux, par la galalithe, la bakélite, le celluloïd, a donné naissance à une tradition de moulage à chaud. À son tour, le plastique allait susciter le développement d'une activité indépendante de la coutellerie : les matières plastiques qui entrent dans la composition des manches de couteaux, servent désormais également à mouler des chaussures, des embouts, des contenants,... Les ateliers 
de forge, d'estampage, de matriçage, de découpage, de traitement de surface, ont ensuite élargi le domaine de leurs compétences au-delà de la coutellerie, tout en utilisant les mêmes machines, à la fabrication de pièces pour l'industrie automobile ou l'armement.

Ces évolutions se sont traduites par une modification considérable de la structure industrielle. Désormais, même si la coutellerie thiernoise assure $70 \%$ de la production nationale de couteaux et exporte environ $20 \%$ de ses fabrications, le Pays de Thiers n'est plus dominé par une mono-activité. Au début des années 90, la coutellerie a cédé la première place aux autres branches de la métallurgie, c'est-à-dire à l'ensemble des activités autrefois sous-traitantes de la coutellerie qui, par voie de dérivation à partir des années 70 , ont élargi leurs domaines de compétence à d'autres secteurs industriels. C'est ainsi que le Pays de Thiers constitue actuellement l'un des premiers pôles français dans le secteur de la forge et de l'estampage, ce qui traduit sans conteste une mutation radicale. Mais cette mutation a été largement soutenue par les politiques publiques.

\subsection{L'action avant-gardiste des acteurs publics locaux}

En effet, le développement précoce d'une mono-activité d'une part, et par voie de conséquence l'identification étroite de la région thiernoise à la région coutelière, la grande endogénéité de la population d'autre part, qui s'est traduite par l'importance exacerbée du caractère familial des entreprises, avaient conduit à un certain enfermement des Thiernois dans ce territoire et cette activité, d'où les difficultés ultérieures d'adaptation à une économie de plus en plus mondialisée, réclamant des échanges nombreux et variés avec l'extérieur du territoire. Si le système productif thiernois pouvait s'appuyer sur un certain nombre d'atouts (sa notoriété, ses savoir-faire, les compétences techniques de sa main-d'œuvre), il présentait aussi, dès les années 70, des signes d'essoufflement, dont les causes sont à rechercher principalement dans cet enfermement territorial et professionnel, qui limitait la diffusion des innovations. C'est pourquoi les principales institutions de la région thiernoise (principalement la Chambre de Commerce et d'Industrie et la municipalité de Thiers), prenant la mesure du risque de sclérose, ont engagé leur action dans deux directions complémentaires : la nécessaire ouverture du système productif et la modernisation de l'identité industrielle (Fournier, 1999). Ce qu'il convient de souligner dans le cas présent, c'est la précocité de cette prise de conscience et surtout des actions qui en ont découlé, mais aussi le caractère avant-gardiste des acteurs publics locaux. Cette fonction «d'avant-garde » (Bloch-Lainé, 1999 ; Parodi, 1998) implique en effet une aptitude à reconnaître les besoins socio-économiques et une capacité à les « révéler » (ibid) au territoire local. Ces acteurs sont des «modernes » dans le sens où ils comprennent les évolutions à venir et tentent d'y adapter le système productif territorialisé.

En effet, à la fin des années 60, pratiquement toutes les entreprises de sous-traitance du Pays de Thiers travaillaient exclusivement pour la coutellerie. Certains chefs d'entreprise, conscients de la fragilité d'un tel système, ont commencé à diversifier leur activité : les forges et le découpage, notamment, se sont tournés vers les secteurs de l'automobile, de l'aéronautique, tandis que les fabricants de manches de couteaux en plastique se sont orientés vers la production de pièces techniques pour l'industrie (automobile, électroménager, emballage...). Si le mouvement de diversification a démarré spontanément, à l'initiative de quelques innovateurs, la C.C.I. de Thiers a largement 
contribué à la diffusion du phénomène. Durant une dizaine d'années, de 1975 à 1985, un important travail de conseil et d'assistance a été mené auprès des sociétés sous-traitantes de la coutellerie, grandes ou petites, pour les engager à élargir leur clientèle vers d'autres secteurs d'activité et d'autres horizons géographiques. Elle les a également incitées à participer à des salons professionnels (Midest surtout) et à des salons internationaux, comme la Foire de Hanovre, en assurant notamment l'organisation de stands collectifs à partir de 1989. Cette action, qui s'est poursuivie sans interruption depuis 1976, représente une part importante de son activité puisqu'elle occupe une personne à temps complet.

Par ailleurs, comme nombre de collectivités locales françaises depuis les lois de décentralisation, les communes du Pays de Thiers ont mis en œuvre un certain nombre d'actions assez classiques pour maintenir et développer l'activité. Mais l'originalité de la Ville de Thiers est d'avoir également mis l'accent sur la modernisation de l'identité thiernoise. Prenant conscience, à partir de 1976, des mutations qui affectent la coutellerie, un ensemble de personnalités, craignant que les progrès techniques ne conduisent à la disparition des savoir-faire traditionnels, commencent à envisager la création d'un lieu de mémoire, de patrimonialisation. Le Musée de la coutellerie verra le jour en 1982. Cependant, le concept de patrimonialisation a été conçu de manière très large -ce qui souligne à nouveau la vision avant-gardiste des Thiernois- puisqu'il s'est aussi agi de sauvegarder des savoir-faire en voie de disparition. C'est pourquoi a été associée au Musée une entreprise de fabrication, la Maison des Couteliers ${ }^{6}$, qui a pour objectif « d'impulser une dynamique à la coutellerie artisanale par la production de couteaux d'art et par l'innovation dans l'emploi de matériaux et de formes nouvelles ». Il s'agissait de former de jeunes artisans à des métiers en voie de disparition, émouleur ou façonneur de manches, et de favoriser le développement de l'artisanat d'art et la diffusion du design. L'enjeu final consistait à hisser l'ensemble de l'activité coutelière vers le haut de gamme.

Ces différentes actions collectives n'ont pas seulement permis de soutenir les mutations du système industriel thiernois. Elles ont également fait émerger, dans le domaine de la sous-traitance, de nouveaux réflexes de solidarité territoriale, de nouvelles synergies entre industriels. Sur les stands collectifs des salons professionnels, on constate une remarquable entraide entre les entreprises des divers secteurs de la sous-traitance, qui se manifeste jusque dans la conquête de nouveaux marchés: les industriels n'hésitent pas à orienter par exemple leurs clients importants vers des confrères thiernois dont l'activité est complémentaire de la leur. Ces actions ont aussi favorisé la construction d'une nouvelle image collective. Désormais, au Midest, les donneurs d'ordre recherchent le pôle thiernois, qui tient à s'affirmer bassin de sous-traitance. L'association «Transformance », qui regroupe toutes les entreprises de sous-traitance du Pays de Thiers, quelle que soit leur spécialité (métallurgie, emballage, maintenance,...) a institutionnalisée cette nouvelle solidarité professionnelle et territorialisée. Ce n'est cependant pas cette spécialité nouvelle qui a été proposée pour l'appel à projets de la Datar, mais le secteur traditionnel de la coutellerie.

${ }^{6}$ Le Musée a un statut municipal alors que la Maison des Couteliers a été conçue dans le cadre d'une association qui rassemble toutes les institutions thiernoises : municipalité, chambres consulaires, syndicats professionnel... 


\subsection{Reconnaissance et poursuite des actions dans le cadre du label SPL}

En 1997, le gouvernement français confiait à la Datar la mission de «favoriser au niveau local la constitution ou la consolidation de réseaux de petites et moyennes entreprises et renforcer les structures locales qui s'y consacrent ». Cet objectif affiché souligne bien une rupture dans la politique industrielle de la France. Son directeur Jean-Louis Guigou considérait que la «France avait pris du retard » dans le domaine de la coopération inter-entreprises en raison du « jacobinisme industriel propre [au] pays, du colbertisme d'entreprise » (Datar-OCDE, 2001). Le modèle de référence explicite de cette nouvelle politique est l'Italie et ses districts, mais la position de la France est beaucoup plus timide : il n'est en aucun cas question de faire appel à la loi pour définir les systèmes productifs locaux (SPL). On se contentera d'un simple appel à projets, doté au final de moyens assez modestes. En 1998 et 1999, la Datar a lancé deux appels à projets et « labellisé » au final 96 SPL, à partir de critères au demeurant assez fluctuants et ambigus, ce qui donne lieu à un certain nombre de confusions entre de véritables SPL qui n'ont pas jugé utile de répondre à l'appel d'offre et des espaces industriels plus classiques qui ont considéré avec intérêt cette opportunité, transformant l'appel d'offre en mesure de labellisation, comme on le verra avec les exemples suivants d'Aurillac et Lacaune ${ }^{7}$.

Lancé le 29 octobre 1998, le SPL de la Coutellerie de Thiers se présente comme un programme opérationnel qui concerne 275 entreprises industrielles et artisanales (pour moitié des couteliers, pour moitié leurs fournisseurs et sous-traitants). Améliorer la compétitivité de l'ensemble des entreprises de la filière en favorisant l'innovation d'une part, les démarches Qualité/Certification d'autre part, rassembler les entreprises pour mener des opérations collectives de promotion et de prospection sur les marchés français et internationaux, accroître la notoriété du bassin coutelier de production de Thiers et ses performances collectives et individuelles en terme d'emplois et de parts de marché constituent les principaux objectifs inscrits au programme du SPL. Celui-ci a démarré par la réalisation d'une double étude cherchant à préciser, d'une part, les données économiques de la filière et, d'autre part, l'état de son marché en Europe et dans le monde. À l'issue de ce diagnostic, plusieurs opérations ont été engagées, qui s'inscrivent pour la plupart dans la lignée de ce que les institutions locales (C.C.I. et municipalité) avaient amorcé il y a près de 30 ans.

\footnotetext{
${ }^{7}$ Dans l'appel à projets lui-même, s'affichaient deux définitions contradictoires. Au bas de la première page, présentant les principes de l'appel à projets, on pouvait lire : "On entend par système productif local (SPL) un ensemble caractérisé par la proximité d'unités productives qui entretiennent entre elles des rapports d'intensité plus ou moins forte. Les rapports des unités entre elles se présentent sous plusieurs formes : formels, informels, matériels, immatériels, marchands et non marchands. La notion de système de production localisé se présente donc comme un ensemble d'activités interdépendantes techniquement et économiquement organisées et territorialement agglomérées ». Il s'agit dans ce cas d'une définition clairement qualitative qui insiste sur la diversité des relations que peuvent entretenir les entreprises au sein d'un territoire, définition qui s'inspire des observations des sociologues italiens ayant travaillé sur les districts de la Troisième Italie. Mais quelques pages plus loin, dans la présentation du cahier des charges, c'est une vision beaucoup plus statistique et rigide qui est retenue pour préciser ce qu'on doit entendre par caractère concentré de l'activité : « une mesure de la concentration est donnée par l'analyse statistique par zones d'emplois (...). Il s'agit de zones géographiques dont l'activité est exercée dans dix établissements productifs au moins, représente $5 \%$ au moins de la zone d'emploi, et $5 \%$ au moins de la branche d'activité en France. ».
} 


\section{Aurillac et Lacaune : de la capacité du local à s'approprier une politique publique ponctuelle}

La politique de «SPLisation» de sites a constitué une opportunité à saisir pour des territoires fragilisés par les évolutions économiques. Ces territoires ne constituent pas forcément de véritables SPL au sens classique, mais leur capacité à se saisir d'une opportunité d'accompagnement au développement met en valeur l'appropriation et la traduction d'une politique publique en fonction des besoins locaux.

Nombre d'ensembles économiques sont fondés sur une tradition ancienne, rappelons-le, cependant, face aux contextes de plus en plus concurrentiels des marchés, leur spécificité territoriale, leur créneau, leur dynamique entrepreneuriale,... ne suffisent plus à assurer leur pérennisation, surtout si les espaces souffrent d'isolement vis-à-vis des grands axes de circulation, comme c'est bien souvent encore le cas en moyenne montagne, notamment à Aurillac et Lacaune. Dans le cadre de ces quasi enclaves économiques, la proximité entre le patronat local et le monde politique local est facteur de développement. De la même façon, les représentations des décideurs publics quant au maintien d'une vitalité économique ${ }^{8}$ dans les espaces fragilisés ont conduit à la reconnaissance plus aisée d'une labellisation SPL. La démarche est ainsi parfois moins basée sur une logique de reconnaissance que sur une perspective d'impulsion en fonction de la capacité du local à s'approprier une appellation (conjoncturellement ?) porteuse. En effet, le Lacaunais et l'Aurillacois ont obtenu l'appellation SPL pour la filière charcuterie, pour le premier, et pour la filière bois, pour le second (Fournier et al, 2003). Cette forme de certification officielle s'ajoute à d'autres opérations d'un " savoir utiliser » l'offre publique pour impulser des adaptations du local, comme ce fut particulièrement le cas en 1995 avec la commande de parapluies d'Aurillac d'un candidat à la présidentielle : les caractéristiques de la commande (grande quantité et délais réduits) obligèrent les derniers fabricants locaux à se constituer en GIE (Groupement d'intérêt économique) ; ce GIE a permis d'améliorer les moyens techniques et commerciaux et, à terme, a réduit l'ampleur du déclin de cette activité.

\subsection{Le SPL et la structuration de la filière bois aurillacoise}

Aurillac est une ville moyenne qui rassemble environ 56000 habitants dans l'agglomération. Le poids de la communauté d'agglomération (CA) est fondamental au sein de l'économie cantalienne : $43 \%$ des emplois du département, pour guère plus que le tiers de la population totale, et $48 \%$ de la valeur ajoutée départementale. Pour des raisons environnementales et de maintien d'une activité traditionnelle, les acteurs publics ont soutenu nombre de démarches de certification et de valorisation du bois, particulièrement pour que les entreprises locales puissent rester dans les fichiers fournisseurs des grandes firmes nord-européennes. Dans cette perspective, la structuration de la filière bois d'Aurillac est le résultat de l'implication de deux acteurs locaux : le dirigeant d'une menuiserie locale et la CA.

8 Notamment à travers le soutien à l'économie locale via les actions de l'ADIMAC, Association pour le Développement Industriel du Massif Central, au sein des Plans Cantal (5 depuis 1990). 
À l'origine, ce sont les besoins d'un dirigeant d'entreprise (Caumon JP menuiserie ${ }^{9}$ ) en bois sec qui lancent le processus. Après enquête auprès des entreprises locales, 107 entreprises se révèlent intéressées par la création d'un séchoir à bois collectif, alors que l'offre locale est largement insuffisante. Dès lors, Caumon a réussi à fédérer les professionnels du bois autour d'un projet commun. L'installation est implantée sur une friche industrielle appartenant à la CA. La gestion en est confiée à une entreprise créée pour l'occasion, la $\mathrm{SA}$ «pôle bois » avec 44 actionnaires. Le projet de près de 1,5 million d'euros est en grande partie financé par la CA, avec des co-financements ADEME, Conseil régional... Les actionnaires versent un loyer annuel, dans le cadre d'un bail de 20 ans, au bout duquel ils deviendront propriétaires de l'ensemble. Par ailleurs, la labellisation SPL a permis l'obtention d'une aide européenne, attribuée par le FNADT ${ }^{10}$, et de subventions de fonctionnement. Cet aménagement, opérationnel depuis 2002, a permis de rapprocher des entrepreneurs sur une action concrète. Il est également un premier moment de sensibilisation de la filière aux questions environnementales, toujours plus présentes dans la législation et auprès des clients de la filière. Pour autant, cette préoccupation n'a pas été le moteur de l'opération.

La «SPLisation » de la filière bois aurillacoise est avant tout le résultat d'une implication des acteurs locaux. La réponse à l'appel d'offre est stratégique. Elle est l'aboutissement d'un besoin entrepreneurial accompagné par les organismes publics locaux, dans la perspective de dynamiser ou tout simplement de pérenniser un secteur d'activité. Dans ce sens, le SPL local n'en est pas véritablement un, selon la définition commune donnée à ce sigle, par contre, les acteurs publics locaux ont véritablement impulsé une dynamique en s'engageant auprès des entrepreneurs de l'agglomération et en inscrivant leur démarche au sein d'une politique publique. De fait, les partenaires, locaux mais aussi nationaux, ont su saisir une opportunité pour bénéficier d'un cadrage et des financements que cela implique. Les objectifs des élus aurillacois sont bien entendu le développement des activités économiques locales, notamment pour compenser un handicap situationnel. En revanche, la capacité à saisir des outils de développement provient à la fois de convictions d'élus et du savoir-faire (montage de dossier, orientation des dossiers, argumentaire,...) de techniciens locaux pour faire entrer le local dans les arcanes de politiques publiques.

\subsection{Le pôle charcutier de Lacaune : la réinvention d'une culture de terroir}

Le cas de Lacaune, en bordure sud du Massif central, dans le département du Tarn, est quelque peu similaire en termes d'appropriation d'une politique. En revanche, Lacaune ne compte guère plus de 3000 habitants. De par sa situation en moyenne montagne, elle s'est depuis longtemps spécialisée dans la charcuterie de type salaisons, au profit, traditionnellement, des bas-pays méditerranéens (Biterrois,...). Depuis quelques décennies ses attaches sont davantage orientées vers Albi et Toulouse, notamment du fait de la régionalisation. Cet espace reste encore relativement isolé ; isolement et qualité de la

\footnotetext{
950 salariés à Arpajon-sur-Cère

${ }^{10}$ FNADT, Fond national d'aménagement et de développement du territoire.
} 
vie (climat, montagne, tranquillité) sont parmi les arguments évoqués par les partenaires locaux pour justifier leurs attaches malgré les difficultés liées à l'éloignement des centres urbains de plus grande taille.

La charcuterie des Monts de Lacaune représente un pôle charcutier important avec 35000 tonnes de produits (soit 240000 porcs traités) en 2003 et plus de 800 emplois directs. À l'échelle de deux cantons, ce secteur d'activité est donc fondamental pour l'autonomie et la pérennité de Lacaune ; cette petite ville demeurant un pôle de services essentiel (collège, poste, commerces...) pour une population active salariée employée à $90 \%$ sur les différentes communes de l'ensemble.

Pendant longtemps, l'économie locale s'est caractérisée par une concurrence forte entre les entreprises locales ; cette concurrence, d'autant plus forte que les productions sont similaires ou très proches, était (et est) avivée par la prégnance du milieu local (histoires inter-familiales). Par ailleurs, l'individualisme est ici renforcé par le parcours professionnel qui permet de passer du statut de revendeur à celui de producteur. Tous ces éléments ont freiné la prise de conscience d'un intérêt commun. C'est l'appréhension de fragilités, en cours et à venir, qui a fait évoluer les mentalités et mobiliser les acteurs. Or, le Lacaunais a connu une certaine marginalisation à laquelle se sont ajoutées des mutations d'ordre sociétal (difficulté à faire venir des cadres, déconsidération du travail charcutier, en lien, notamment, avec sa fatigabilité).

La fragilisation sectorielle a provoqué la remise en cause d'un modèle géographique « équilibré » (emploi, habitat,...) où l'espace local assurait toutes les fonctions de la vie quotidienne. Cette situation a même été survalorisée par le type de production : le secteur charcutier local a longtemps été perçu comme sans problème, par la logique de l'évidence (« on a toujours mangé du saucisson en France »). La deuxième moitié des années 80 est venue briser cette représentation. Plusieurs entreprises, relativement importantes (donc considérées comme solides), ont fait faillite. Surtout, ces évolutions ont modifié la base économique : des investissements " étrangers ${ }^{11}$ ont été réalisés. Ils ont concerné des entreprises en difficulté momentanée ${ }^{12}$ ou des créneaux inexploités localement (ateliers de découpe,...). Ces réalités trouvent bien souvent leur origine dans une incapacité à évoluer, liée, par exemple, au contrôle de l'innovation par le jeu fermé des producteurs locaux. Enfin, ces investissements se sont partiellement traduits par des délocalisations, notamment sur Castres et Albi. Ces entrepreneurs recherchaient de plus vastes espaces plans mais aussi des sites mieux desservis.

En résumé, l'espace lacaunais connaît un déclin (démographique, commercial,...) et la perpétuation de cette mono-activité est apparue comme vitale auprès des décideurs locaux. La proximité des fonctions politique et économique est ici nette : les maires ou les principaux adjoints sont des charcutiers et la charcuterie est l'enjeu commun ${ }^{13}$ de développement. Cependant, les charcutiers lacaunais sont essentiellement des producteurs de charcuterie générique, souvent réalisée en sous-traitance pour des marques de grandes surfaces, ou bien commercialisée avec une marque propre à l'entreprise mais sans affichage de la ville et de la région (Monts de Lacaune).

\footnotetext{
${ }^{11}$ Est considéré peu ou prou comme étranger tout ce qui n'appartient pas à l'espace lacaunais.

${ }^{12}$ Les faillites ont touché des entreprises économiquement saines mais fragilisées momentanément (successions, trésorerie...).

${ }^{13}$ Même si le tourisme et la production d'eau minérale ne sont pas à négliger.
} 
La prise de conscience, dès les années 80, a débouché sur une implication des acteurs professionnels (Midiporc), territoriaux (Conseils général et régional) et locaux non seulement pour le montage de dossiers mais également autour de la quasi réinvention d'une culture locale autour du cochon et de la montagne. Une fête du cochon est préparée en juin depuis 1990, un concours du meilleur jambon cru a été lancé, un circuit de la salaison de Lacaune a été mis en place et une maison de la charcuterie, retraçant l'histoire et l'importance de cette activité, s'est ouverte. Par ailleurs, une démarche d'obtention d'une AOC « Jambon de Lacaune » est initiée depuis 1992. Midiporc, l'association des producteurs de salaisons,... ont participé à construire un produit (charte, traçabilité, durée d'affinage,...) pour associer un produit (de qualité) à un terroir. C'est dans ce cadre que les acteurs ont inscrit leur démarche à l'appel d'offre SPL, dans un souci de reconnaissance et d'accélération de la procédure Toutefois, à ce jour, le processus de reconnaissance officielle se heurte à un problème de taille : le Lacaunais ne produit pas de porcs, élément nécessaire à la territorialisation du produit. Une des solutions possibles semble résider dans la production de porcelets nés, « en élevages AOC», sur les espaces voisins (Aveyron, Lot,...). De fait, le lobbying des acteurs a pour objectif le «terroir » de fabrication pour maintenir coûte que coûte une production en montagne.

\section{Benedita : de la création ex nihilo d'un développement local à une territorialisation à risques}

Le développement local est un élément du vivant. Dans certains cas, il mute ou disparaît, dans d'autres, il naît en grande partie de la volonté des pouvoirs publics. La période contemporaine a vu la naissance de tels espaces, lancés par les acteurs politiques en raison de la richesse du terreau local. C'est actuellement le cas de Benedita (Portugal), exemplaire de 40 ans de métamorphose du local.

Benedita est une petite ville de 8500 habitants située à une centaine de kilomètres au nord de Lisbonne. Elle est aujourd'hui un centre industriel ${ }^{14}$ composé en grande majorité de très petites entreprises ( $70 \%$ des 200 entreprises), fréquemment familiales. Le secteur secondaire se concentre autour de quelques secteurs d'activité : le cuir et la maroquinerie, la coutellerie et l'extraction et le travail de la pierre ${ }^{15}$. La société d'interconnaissance, caractéristique de Benedita, permet la multiplication des échanges, donc l'établissement de la confiance, nécessaire à la dynamique économique locale. Ces relations sont formelles et informelles; naturellement, les firmes s'échangent des biens et des services ; fréquemment elles organisent aussi des systèmes de sous-traitance en chaîne qui leur permet l'accès à des marchés importants, supérieurs à leurs capacités individuelles. Mais les échanges sont également informels, entre les industriels d'une part, qui déjeunent souvent $\mathrm{au}$ « Bar des entrepreneurs » au centre de Benedita, entre les chefs d'entreprises et les salariés d'autre part, qui se rencontrent aussi fréquemment en dehors des lieux de travail (vies associative et religieuse).

Si le tissu industriel de Benedita présente les caractéristiques désormais classiques des systèmes productifs localisés, sa genèse révèle une certaine originalité. Non seule-

\footnotetext{
${ }^{14} 60 \%$ de la population active travaille dans l'industrie.

${ }^{15}$ Provenant du massif calcaire sur lequel est adossée la ville, la Serra dos Candeeiros.
} 
ment l'industrialisation est ici assez récente (pratiquement aucune entreprise n'a été créée avant 1960), mais elle s'inscrit aussi en rupture avec les traditions locales : les petites villes voisines d'Alcobaça et Caldas da Rainha constituaient d'importants centres traditionnels de fabrication de céramique. Le système productif de Benedita n'est donc en rien l'héritier de la proto-industrie ; il s'ajoute à la liste déjà longue, mais moins connue de ceux qui, en Europe, ont émergé et se sont affirmés dans l'après guerre (Houssel, 1995 et 2000; Fournier, 2002). L'émergence d'une dynamique industrielle à Benedita est le résultat d'une douzaine d'années d'actions de développement local, d'animation du milieu local, réalisées tout d'abord de façon empirique, puis de plus en plus institutionnalisées. Entre le début des années 50 et le milieu des années 60 , se sont cumulés nombre d'événements, de démarches de développement local, à la réussite desquels Benedita doit son expansion. L'articulation diachronique de ces faits est donc importante pour comprendre comment a pu s'engager la dynamique de développement.

Tout commence dans les années 50 par l'action d'un prêtre novateur qui a mis en œuvre une stratégie « empirique » pour favoriser le lien social. Soucieux d'améliorer la communication et la solidarité entre ses paroissiens dans cette région d'habitat dispersé (Benedita compte plus de trente hameaux), il les a convaincus de reconstruire ensemble (1952-1955) et avec leurs deniers une nouvelle église, pour remplacer l'ancienne, jugée trop petite. Cette réalisation a supposé la création d'un certain nombre d'associations, de comités pour récolter les fonds nécessaires, et de groupes de travail pour la réaliser.

Dans les années 60, cette première expérience d'action collective a certainement permis à la freguesia d'être choisie comme site expérimental dans un programme national de Développement Communautaire (1962). Certes, la situation politique est très particulière : la dictature interdit l'émigration alors que le travail est si rare. Au plan économique, cette action a consisté à créer des entreprises coopératives en rassemblant les artisans existants, afin de favoriser la mécanisation, d'augmenter la production et la rentabilité, pour pérenniser ou créer des emplois. Plusieurs entreprises coopératives vont voir le jour dans le domaine de la chaussure et de la coutellerie et vont rapidement créer des emplois. La création, en 1963, d'un collège coopératif (Cooperativa de Ensino e Cultura) a joué un grand rôle tant pour le développement de Benedita que pour la constitution d'un fort sentiment d'appartenance. L'existence du collège constitue un maillon essentiel de la dynamique locale. En élevant le niveau de formation générale de la population, le collège a permis de conforter la dynamique entrepreneuriale. Enfin, un événement totalement extérieur, l'ouverture, en 1964, de la route nationale (RN1) reliant Lisbonne à Porto, qui traverse Benedita, va venir conforter la dynamique industrielle naissante en favorisant les échanges avec les grandes villes. Cette première période de coagulation des forces va durer une dizaine d'années. Très rapidement, à cette première phase de coopération a succédé une phase de fragmentation (Rufino, 1996), qui a permis la diffusion du processus industriel ; on peut illustrer le phénomène avec l'exemple d'une entreprise de maroquinerie, fondée en 1969 avec 11 associés : 9 d'entre eux ont progressivement quitté la société initiale pour créer leur propre affaire.

À Benedita, les champs de l'économique, du culturel et du politique sont étroitement mêlés : la Junta de Freguesia n'est-elle pas installée dans une ancienne usine de chaussures ? Les modalités de la dynamique industrielle et le processus de développement local qu'a connu Benedita au cours des décennies passées ont conduit à consolider, voire 
à construire, une identité qui se vit en rupture avec les territoires voisins, en particulier avec le siège du Municipe, Alcobaça. La réussite économique s'est peu à peu traduite par un besoin de reconnaissance, une revendication d'autonomie au plan politique (ChignierRiboulon et Fournier, 2003), qui se fonde autant sur ces représentations identitaires que sur les besoins concrets des industriels et de la population. Par ailleurs, la mobilisation citoyenne, née autour du catholicisme et du développement local, se manifeste quotidiennement, aujourd'hui encore. L'action associative des Beneditains répond tout d'abord à certaines carences de l'État. Cette politique sociale correspond à l'environnement mental local qui associe une collaboration commune autour d'un projet (d'entreprise ou d'association) puis, lorsque la réalisation fonctionne correctement, la logique individuelle est privilégiée au nom de l'objectif de réussite individuelle. Enfin, cette solidarité sociochrétienne s'exerce avant tout au profit de la « communauté beneditaine », au nom du lien commun, mais probablement aussi dans un souci de cohésion présente et future.

Les auteurs, socio-psychiatres ou ethno-psychiatres (Malewska-Peyre et Tap, 1993 ; Devereux, 1973), qui ont travaillé sur l'image de soi soulignent l'importance de la recherche d'une identité positive. Avant la réussite manifeste de Benedita, les habitants des freguesias environnantes considéraient les Beneditains avec un certain mépris, car ils étaient les plus pauvres et les plus attachés à une agriculture de subsistance; or, la valorisation, individuelle et collective, a été très porteuse, socialement et économiquement : le « je suis de Benedita » est affirmé avec force. Il montre une identification forte à l'espace et un attachement certain à la ville. Il est aussi l'expression d'un orgueil, celui issu de la force du travail, du mérite lié à une réussite inespérée une ou deux générations avant. Ce vécu est à rattacher au concept de résilience décrit par Boris Cyrulnik (1999) que l'on a pu repèrer en d'autres lieux (Fournier, 2000). Cependant, c'est aussi un processus localiste (Gontcharoff, 1999) dans le sens où il est fondé sur un entre-soi qui tend à exclure les autres. Dans cette perspective, le risque peut être à terme l'auto-satisfaction, l'enfermement, une solidarité réduite au groupe de la freguesia... et l'amorce d'un déclin économique.

\section{Conclusion}

Au-delà de la diversité observée, l'un des enjeux des espaces économiques territorialisés sera de préserver les caractéristiques sociales et culturelles, qui ont jusqu'alors constitué le ressort intérieur de leur dynamique de développement, tout en restant ouverts aux influences extérieures, afin de s'adapter à la mondialisation de l'économie et de continuer à être « d'ici et du monde ». Si le risque d'enfermement est parfois réel, la pérennisation d'une certaine forme d'identité locale ne sera pas forcément dans l'avenir le plus facile à réaliser, tout du moins dans les pays développés. En effet, même si l'on veut bien tenir avec Guy Di Méo (1998) « le pari de la résistance des territoires », force est de constater que pour continuer à occuper des créneaux spécifiques, les systèmes productifs territorialisés ont besoin de compétences techniques de plus en plus pointues, acquises plutôt dans des instituts de formation spécialisés que « sur le tas », dans les ateliers, au contact de la tradition; or, les psychosociologues commencent à démontrer qu'avec l'élévation du niveau socio-culturel, la rationalité progresse. «Le mondialisme technique dilue le sentiment d'appartenance, (...) pulvérise le lien social, (...) désaffective le monde », écrit Boris Cyrulnik (1997). À l'heure de la concurrence mondialisée, les territoires ne peuvent 
plus seulement s'appuyer sur les dynamiques entrepreneuriales endogènes, les valeurs et savoir-faire issus des lieux. Ils ont de plus en plus besoin que les institutions en place remplissent des fonctions auxiliaires toujours plus complexes, qui vont de l'amélioration de l'environnement technique des PMI (l'agence Lumetel) à la culture des valeurs et des idéologies territoriales, par le biais, notamment, de la patrimonialisation des savoir-faire (Thiers, Lacaune), voire à l'invention et à la diffusion, par tous les moyens de communication disponibles, de nouveaux mythes destinés à les rendre « désirables » pour les créateurs d'entreprises comme pour leurs salariés.

\section{Références}

Becattini G., 1989, Riflessioni sul distretto industriale marshalliano como concetto socio-economico, Stato $e$ Mercato, $\mathrm{n}^{\circ} 25$

Benko G., Lipietz A. et al., 1992, Les régions qui gagnent, Districts et réseaux : les nouveaux paradigmes de la géographie économique, Paris, 424 pages.

Benko G., 2000, La richesse des régions : la nouvelle géographie sociale et économique mondiale, Paris, PUF, 564 pages.

Bloch-Laine F., 1999, Faire société : les associations au cœur du social, Paris, Syros.

Chignier-Riboulon F., Fournier M., 2003, Emergence et affirmation d'une petite ville industrielle : Beneditta Portugal, in L'avenir des petites villes, CERAMAC, Clermont-Ferrand, pages 175-190.

Cyrulnik B., 1997, , L'ensorcellement du monde, éd. Odile Jacob, Paris, 304 pages.

Cyrulnik B., 1999, Un merveilleux malheur, éd. Odile Jacob, Paris, 240 pages.

Datar, 2001, Réseaux d'entreprises et territoires, Regards sur les systèmes productifs locaux, La Documentation française, Paris, 181 pages.

Datar-Ocde, 2001, Actes du Congrès mondial des systèmes productifs locaux, Paris, 216 pages.

Devereux G., 1973, Essais d'ethno-psychiatrie générale, Paris, Gallimard, 394 pages.

Di Meo G., 1998, Géographie sociale et territoires, Paris, Nathan, 310 pages.

Fournier M., 1999, « Le système productif thiernois en 1999: rôle des institutions locales et résilience », in Dany Hadjadj éd, Pays de Thiers, le regard et la mémoire, Presses Universitaires Blaise Pascal, ClermontFerrand, pp. 555-567.

Fournier M., 2000, « De la résilience sigolénoise à la diffusion yssingelaise : création d'entreprise et vitalité des systèmes productifs locaux », in «Les territoires des PME », Revue de l'Economie Méridionale, vol. $48,1-2 / 2000, n^{\circ} 189-190$.

Fournier M., 2002, « Les montagnes-ateliers : un modèle endogène de développement durable », in Bordessoule E, Les Montagnes, Editions du Temps, Nantes, pages 129-151.

Fournier M. et al., 2003, Territoire et environnement des PMI, étude comparée des systèmes productifs des moyennes montagnes européennes, rapport pour le Comité Interrégional de massif/Datar Massif central, 103 pages.

Gontcharoff G., 1999, Valeur et limites du « local », Economie et humanisme, octobre 1999, p 12-17.

Houssel J-P. et al., 1995, Les districts industriels : tradition et innovation, Revue de géographie de Lyon, volume $70, n^{\circ} 1,75$ pages.

Houssel J-P., 2000, L'industrialisation endogène des milieux ruraux de la façade atlantique du Centre-Portugal à la Bretagne, in Les nouveaux espaces ruraux de l'Europe Atlantique, Maison des sciences de l'Homme et de la Société, Poitiers, pages 321-339. 
Malewska-Peyre H, Les troubles de la socialisation chez les jeunes issus de l'immigration, in Hanna MalewskaPeyre et Pierre Tap, 1993, Marginalités et troubles de la socialisation, Paris, PUF, pages 109-129.

Parodi M., 1998, Sciences sociales et « spécificités méritoires » des associations, Revue du MAUSS, Une seule solution, l'association ? Socio-économie du fait associatif, ${ }^{\circ} 11$, pp 136-154.

Rufino I., 1996, Empresas e Empresarios: Cultura de Empresa a Benedita, tese de mestrado, ISCTE, Lisbonne.

Vidal F., 1998, L'histoire industrielle de l'Italie, de 1860 à nos jours, Seli Arslan, Paris, 256 pages. 
